\title{
Thinking about the thermostat
}

A human that gets too cold can grab a sweater or a blanket; if they get too hot, layers can be shed. Animals don't quite have that luxury, or the ability to control their thermostat. But, ambient temperature can influence physiological variables like metabolism, even though an animal may seem healthy and content. A growing body of literature suggests that lab temperatures for mice might be on the cold side, with sometimes drastic impacts on scientific outcomes as a result. A new Technology Feature considers the temperature variable in biomedical research, and some of the ways that it can be controlled.

See 'Out of the cold' by Esther Landhuis

\section{Pet policy}

Veterinary medicine is always looking to develop new drugs and treatments for animal patients. When something promising emerges that might soon be ready for the clinic, veterinarians will conduct trials that often involve pet animals. The May Protocol Review asks how much information an IACUC needs to assess such trials, and where standard veterinary care ends and its oversight begins.

See 'Protocols for pets: what authority does an IACUC have?' by Jerald Silverman

Published online: 23 April 2018

https://doi.org/10.1038/s41684-018-0064-1

\section{Editorial Board}

Leanne Alworth, DVM, MS, DACLAM

Assistant Director/Attending Veterinarian,

University of Georgia, Athens, GA

Jeffrey Everitt, DVM

Department of Pathology, Duke University, Durham, NC

Craig Fletcher, DVM, PhD, DACLAM

Director, Division of Laboratory Animal Medicine

Assistant Dean for Animal Research Resources

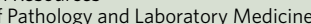

University of North Carolina, Chapel Hill, NC

\section{Charmaine Foltz, DVM, DACLAM}

Director, Division of Veterinary Resources, NIH, Bethesda, MD

\section{Paul Houghton}

CEO, Biologist, Primate Products, Redwood City, CA

Robert F. Hoyt, Jr., DVM, MS, DACLAM

Animal Program Director, Frederick National Laboratory for Cancer Research

National Cancer Institute, Bethesda, MD

\section{Mary Lou James, BA, RLATC}

Consultant, Regulatory Compliance, St. Louis, MO

Alicia Z. Karas, DVM, MS, DACVA

Assistant Professor, Anesthesia, Department of Clinical Sciences,

Cummings School of Veterinary Medicine, Tufts University, North Grafton, MA

Bruce W. Kennedy, MS, RLATG, CMAR, CPIA

IACUC Administrator, Office of Research, Chapman University, Orange, CA

Krishnan Kolappaswamy, DVM, PhD, DACLAM

Associate Director, Laboratory Animal Medicine-North America Chair, IACUC, Envigo,
Indianapolis, IN

\section{Max Lang, DVM, DACLAM}

Professor and Chairman, Department of Comparative Medicine,

Milton S. Hershey Medical Center, Pennsylvania State University, Hershey, PA

Richard H. Latt, DVM, DACLAM

President, Mispro Biotech Services Inc., Montreal, Quebec, Canada

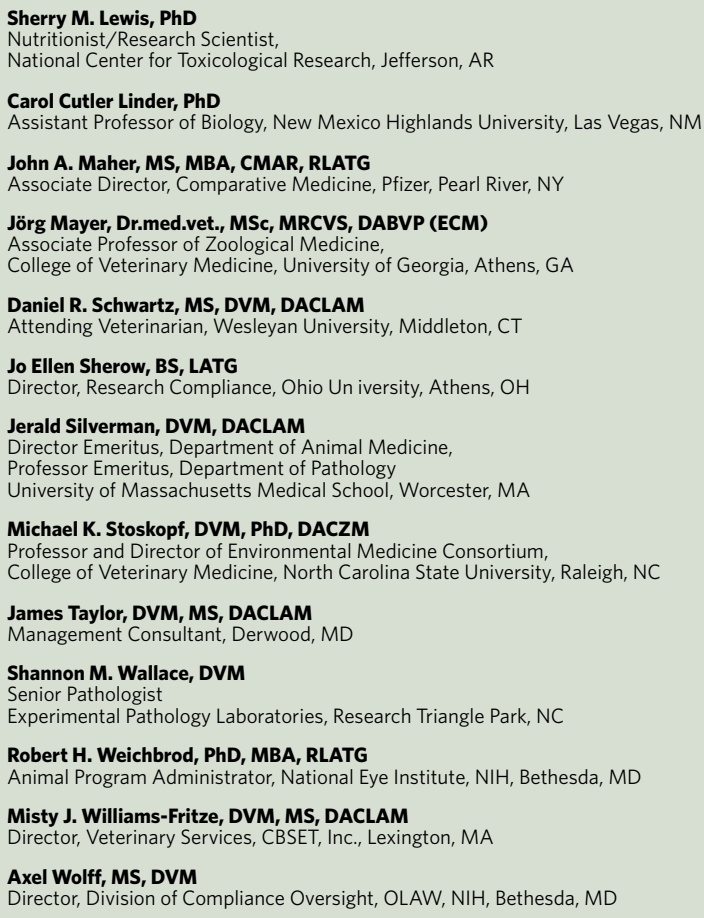

